\title{
Detection of BCR/ABL Fusion Gene by Hematological and Cytogenetical Analysis in Chronic Myeloid Leukemia Patients in Quetta, Pakistan
}

\author{
Bibi Tahira ${ }^{1}$, Muhammad Asif ${ }^{1,2}$, Samiullah Khan ${ }^{1}$,Abrar Hussain ${ }^{1}$, Muhammad \\ Naeem Shahwani ${ }^{1}$, Arif Malik ${ }^{3}$, Syed Inayatullah ${ }^{1}$, Zafar Iqbal ${ }^{4}$, Mahmood \\ Rasool $^{5}$
}

\begin{abstract}
Background: Chronic myeloid leukemia (CML) is a myeloproliferative disorder of pluripotent stem cells, caused by reciprocal translocation between the long arms of chromosomes 9 and $22, t(9 ; 22)(q 34 ; q 11)$, known as the Philadelphia chromosome. Materials and Methods: A total of 51 CML patients were recruited in this study. Complete blood counts of all CML patients were performed to find out their total leukocytes, hemoglobin and platelets. FISH was performed for the detection of BCR-ABL fusion and cryptogenic tests using bone marrow samples were performed for the conformation of $\mathrm{Ph}(9 ; 22)(\mathrm{q} 34 ; \mathrm{q} 11)$ and variant translocation mechanisms. Results: In cytogenetic analysis we observed that out of 51 CML patients $40(88.9 \%)$ were $\mathrm{Ph}$ positive and 4 $(8.88 \%)$ had Ph negative chromosomes. Mean values of WBC $134.510^{3} / \mu \mathrm{l}$, hemoglobin $10.44 \mathrm{mg} / \mathrm{dl}$, and platelets 288.6 10 $3 / \mu \mathrm{l}$ were observed in this study. Conclusions: In this study, $\mathrm{Ph}$ positive translocation between chromosome $(9: 22)(q 34 ; q 11)$ were observed in $40(88.9 \%)$ CML patients.
\end{abstract}

Keywords: Chronic myeloid leukemia - ph chromosome - variant translocation - matinib mesylate

Asian Pac J Cancer Prev, 16 (9), 3793-3797

\section{Introduction}

Chronic myeloid leukemia (CML) is a disease of clonal hematopoietic stem cell, caused by a balanced translocation between the long arms of chromosomes 9 and $22, \mathrm{t}(9 ; 22)(\mathrm{q} 13 ; \mathrm{q} 11)$, known as the Philadelphia chromosome (Ph) (Chavan et al., 2006; Aguayo et al., 2008; Au et al., 2009). It involves myeloid, monocytic, megakaryocytic, B-lymphoid, erythroid, an t-lymphoid linkages (Faderl et al., 1999). The dysfunctional hybrid protein (PBCR-ABL) produced by the chimeric gene $b c r-a b l$ has tyrosine kinase activity, leading to a factor-independent myeloid proliferation and leukemic transformation (Cowan-Jacob et al., 2007; Aguayo et al., 2008; Usmani et al., 2009; Ye et al., 2014).

The translocation of chromosomes 9 and 22 is observed in $90-95 \%$ of CML cases and variant/complex translocation is found around 5-8\% CML patients, in these cases an additional third, fourth or fifth chromosomes are involved with 9 and 22 (Sessarego et al., 2000; La Starza et al., 2002; Achkar et al., 2010).
Imatinib mesylate (IM), is an inhibitor of BCR-ABL protein tyrosine kinase, is a significant orally therapy $\mathrm{CML}$ treatment because this drug is able to induce a complete cytogenetic response in 65-90\% CML patients. Imatinib mesylate blocks proliferation and induces apoptosis of BCR-ABL expressing in CML and AML cells. This drug has met significant success in terms of both prolonged survival and improved quality of life (Cohen et al., 2002a; Cortes et al., 2003; Al-Achkar et al., 2013; Koshiyama et al., 2013; Al-Jamal et al., 2014).

Monitoring of CML patients receiving Imatinib mesylate therapy is commonly done by cytogenetic test and fluorescence in situ hybridization for $\mathrm{BCR}-\mathrm{ABL}$ detection. Cytogenetic analysis is done by performing the G-banding technique. Marrow specimens a examined on direct or short-term (24-hour) cultures at least 20 metaphases were analyzed per sample. Cytogenetic analysis reveals the $\mathrm{Ph}$ chromosome in 90 percent of patients with CML. It was helpful in demonstrating additional karyotypic abnormalities (clonal evolution). Fluorescence in situ hybridization allows analysis of both

${ }^{1}$ Department of Biotechnology, ${ }^{2}$ Office of Research Innovation and Commercialization, Balochistan University of Information Technology, Engineering and Management Sciences, Quetta, ${ }^{3}$ Institute of Molecular Biology and Biotechnology, The University of Lahore, Lahore, Pakistan, ${ }^{4}$ College of Applied Medical Sciences, King Saud Bin Abdulaziz University for Health Sciences, National Guards Health Affairs, Riyadh, ${ }^{5}$ Center of Excellence in Genomic Medicine Research (CEGMR), King Abdulaziz University, Jeddah, Saudi Arabia. Saudi Arabia*For correspondence: asifjallali@yahoo.com 
cells in metaphase and non dividing cells in interphase, and the results are easily quantifiable. Peripheral-blood specimens can be analyzed by interphase fluorescence in situ hybridization. It is fast then conventional cytogenetic test that allows analysis of more cells in metaphase, interphase, and was reliable in assessing cytogenetic responses in CML (Jabbour et al., 2009).

The major signs and symptoms of CML were tiredness, sometimes bleeding, skin lesions, yellowish color of eyes, skin, nails, night sweats, sudden weight loss, high fever, bone pain, abdominal pain, anemia, bruising spots all over the body, shortness of breath, feeling exhausted, burning sensations under feet, hands along with numbness, abnormal blood count and sometimes inflammation of the skin is very obvious in CML patients (Faderl et al., 1999; Sawyers, 1999).

The aim of our study was to get all necessary information about recently diagnosed disease of CML through application of advanced analytical medical tests such as cytogenetic and FISH techniques and possible adverse effects of CML on different organs of the body and blood parameters.

\section{Materials and Methods}

The data about patients of chronic myeloid leukemia was collected from Sandmen Provincial Hospital, CENAR Hospital and Bolan Medical College, Quetta, Pakistan. The research work conducted between the periods of January 2010 to August 2013. Total 51 CML patients, 22 males and 29 females, were recruited in this study. Written informed consent was taken from all individuals participating in this study. The study was also approved from ethical committee of Balochistan University of information technology, engineering and management sciences (BUITEMS).

\section{Data collection}

A questionnaire was preparing on the basis of signs and symptoms of CML patients and filled in by each with help of a qualified physician.

Mostly CML patients were from Quetta city, while few were from other areas of Baluchistan.

$7 \mathrm{ml}$ venous blood was taken from patient with the help of syringe then $2 \mathrm{ml}$ ( 3 to $4 \mathrm{mg}$ ) EDTA was dissolved in it then $1.8 \mathrm{ml}$ of this blood was dissolved in $0.2 \mathrm{ml},(3.2$ $\mathrm{ml}$ ) of sodium citrate. Then the remaining blood was put into tube for coagulation from which the serum was taken as sample for test.

Bone marrow sample was taken from a bone marrow aspiration or by biopsy. The samples were taken from the back of pelvic or (hip bone) of the patients, in some patients it was taken other bones of the patients. For BM aspiration the patients were advised to lie down on bed. Once the area was cleaned, the skin over the hip and bone surface was numbed with local anesthetic. Then a thin hollow needle fit to the tip of the syringe inserted into bone and the syringe suck out a small amount of liquid bone marrow. After removal of BM, biopsy is done. In this test a large needle was pushed down into the bone and the small section of BM was removed, it also causes pain. The sample were send to lab where the look them by microscope to look for Leukemia cells.

\section{$C B C$ laboratory test}

White blood cells (WBC), red blood cells (RBC), hemoglobin $(\mathrm{Hb})$ Platelets, were measured within 1-2 hours of blood sampling by using Nihon Codon fully automatic Hematological analyzer, Japan. CML patients mostly had a large number of WBC Cells. Some patients had a low number of RBC and Platelets. These findings ensured the patients were suffering from leukemia or not and requirement regarding further investigation.

\section{Procedure of cytogenetic test}

Cytogenetic analysis was performed by bone marrow culture using standard technique (Claussen et al., 2002). 20 GTG banded unstimulated bone marrow metaphase cells were analyzed. Karyotypes were expressed by the International System for Human Cytogenetic Nomenclature (Simons et al., 2013)and $\mathrm{Ph}$ was determined (which is diagnosis of CML). Cytogenetic response was calculated based on the prevalence of $\mathrm{Ph}+$ metaphases among at least 20 metaphase cells in each bone marrow sample and was defined as complete $(0 \% \mathrm{Ph}+$ cells $)$, partial (1-5cells), minor (10 Cells).

\section{Procedure of FISH test}

FISH was performed using directly labeled dual color LSI/CEP probes following the probe supplier's instructions (BCR/ABL-Oncor Ventana Medical Systems, Tucson, AZ, USA) for detection of BCR/ ABL. 500 metaphase or interphase cells were counted to produce a BCR-ABL percentage. The main steps in the methodology include hypotonic treatment, fixation of direct demecolcine treated cultures, followed by dehydration, codenaturation, overnight hybridization, washing and mounting of slides in DAPI-antifade. The slides were viewed on a Zeiss Axioskop 2 microscope with a HBO 100 mercury lamp. The slides were analyzed through a fluorescent microscope (Olympus BMX60) with DAPI, rhodamine, FITC and triple band pass filters (Chromo Technology). Slides were showing fluorescent dots where Red dots (rhodamine) corresponded to the ABL (9q34.1) gene and green dots (fluorescein) to the BCR (22q11) gene, so when one cell with two isolated orange and green dots was seen, it was counted as normal, without rearrangement. When a cell with one isolated orange dot, one isolated green dot, one fused orange and green signal was seen, this was considered as presenting irregular rearrangement of CML. Bone marrow studies, including morphological, cytogenetic and fluorescent in situ hybridization (FISH) analysis were performed every 6 months in the first year of study.

\section{Ultrasound of abdomen}

To estimate the organ damaged by the CML disease, the organs of the patients were examined with the help of ultrasonography. The patients spleen, liver kidney, pancreas, gallbladder, and other organs were examined through ultrasound and images were generated which gave the sizes, location and nature of the organs. 


\section{Results}

A total of 51 patients diagnosed with CML were recruited in this study, out of which 22 were male and 29 were female between the age group 6 to 77 years. The median age was 25 to 35 years. Results showed that median age group between 25-35 years $(21.56 \%)$ were more affected. The old age people $(19.56 \%)$ were also suffering from CML. All CML patients were treated with Imatinib Mesylate. We observed that this medicine is successful to some extent in treatment for CML patients. This treatment is considered to be a first choice for CML patients at all stages. Table 1 also shows that illiterate $31(60.78 \%)$ and lower class CML patients were more

Table 1. Physical and Biochemical Characteristics of CML Patients

\begin{tabular}{|c|c|}
\hline Characteristics & $\begin{array}{c}\text { Number and Percentage } \\
(\%)\end{array}$ \\
\hline \multicolumn{2}{|l|}{ Sex } \\
\hline Male & $22(43.13 \%)$ \\
\hline Female & $29(56.83 \%)$ \\
\hline \multicolumn{2}{|l|}{ Age } \\
\hline $0-15$ & $02(3.91 \%)$ \\
\hline $16-25$ & $10(19.60 \%)$ \\
\hline $26-35$ & $11(21.56 \%)$ \\
\hline $36-45$ & $8(15.68 \%)$ \\
\hline $46-55$ & $10(19.60 \%)$ \\
\hline 56 and above & $10(19.60 \%)$ \\
\hline \multicolumn{2}{|l|}{ Education } \\
\hline Illiterate & $31(60.78 \%)$ \\
\hline Literate & $20(39.21 \%)$ \\
\hline \multicolumn{2}{|l|}{ Location } \\
\hline Urban & $28(54.90 \%)$ \\
\hline Rural & $23(45.09 \%)$ \\
\hline \multicolumn{2}{|l|}{ Socio-economic status } \\
\hline Upper class & $11(21.56 \%)$ \\
\hline Middle class & $18(35.29 \%)$ \\
\hline Lower class & $22(43.13 \%)$ \\
\hline \multicolumn{2}{|l|}{ Smoking status } \\
\hline Smokers & $24(47.05 \%)$ \\
\hline Non Smokers & $27(52.94 \%)$ \\
\hline \multicolumn{2}{|l|}{ Cryptogenic Test $(n=45)$} \\
\hline $\mathrm{Ph}(+)(9 ; 22)(\mathrm{q} 34 ; \mathrm{q} 11)$ & $41(91.11 \%)$ \\
\hline $\mathrm{Ph}(-)(9 ; 22)(\mathrm{q} 34 ; \mathrm{q} 11)$ & $4(8.88 \%)$ \\
\hline \multicolumn{2}{|l|}{ CML Phase at Diagnosis } \\
\hline Chronic Phase & $43(84.31 \%)$ \\
\hline Accelerated Phase & $4(7.84 \%)$ \\
\hline \multicolumn{2}{|l|}{ FISH (27\51) } \\
\hline Bcr-abl (+) & $23(85.18 \%)$ \\
\hline Bcr-abl (+) & $04(14.81 \%)$ \\
\hline \multicolumn{2}{|l|}{ Treatment } \\
\hline Imatinib mesylate (Glevec) & $49(96.07 \%)$ \\
\hline Other & $2(3.92 \%)$ \\
\hline Hematological Analysis & \\
\hline$<1110^{3} / \mathrm{ul}$ & $51 / 04(7.01 \%)$ \\
\hline$>1110^{3} / \mathrm{ul}$ & $51 / 47(92.15 \%)$ \\
\hline \multicolumn{2}{|l|}{ Hemoglobin } \\
\hline$<12 \mathrm{mg} / \mathrm{dl}$ & $51 / 34(66.66 \%)$ \\
\hline$>12 \mathrm{mg} / \mathrm{dl}$ & $51 / 17(33.33 \%)$ \\
\hline \multicolumn{2}{|l|}{ Platelets } \\
\hline$<40010^{3} / \mathrm{ul}$ & $51 / 16(31.37 \%)$ \\
\hline$>40010^{3} / \mathrm{ul}$ & $51 / 35(68.62 \%)$ \\
\hline
\end{tabular}

effected from this disease.

Among the 51 enrolled patients 43 (84.31\%) CML patients were in chromic and $4(7.84 \%)$ were in accelerated phase. We have recently treated 51 patients in which 29 CML patients started their initial therapy with daily doses of 400-600mglday.

Cytogenetic test of CML patients were performed for the detection of the $\mathrm{Ph}$ positive and $\mathrm{Ph}$ negative cells in Patients. The Cytogenetic test of $45 \mathrm{CML}$ patients was performed, in which $41 \mathrm{CML}$ patients had $\mathrm{Ph}$ positive and 4 patient's shows negative results.

FISH test of $27 \mathrm{CML}$ patients were performed in which $23(85.18 \%)$ CML patients showed positive BCR-ABL translocations. BCR-ABL translocations were noted in 500 nuclei cells. It was performed by dual color orange and green, dual fusion translocation probes which are hybridized in interphase nuclei of CML patient. Precision after incorrect numbers of spots were rejected, the precision of spots were detected from $60-98 \%$ in 500 nuclei cells in $23 \mathrm{CML}$ patients which shows positive BCR-ABL translocations. Positive and negative cells were rejected based on orange and green FISH spots.

Hematological test of all 51 patients were performed. We observed that only four patients of CML have normal WBC counts 4-11 10\%3l and 47 (92.15\%) CML patients showed induced values of WBC. In this study normal PLTs count 1500-400 103/ul were observed in 16 CML patients. Anemic CML patients around 34 were investigated out of 51 CML cases.

Table 2 presents different symptoms which were found in CML patients. Due to these symptoms the patients were diagnosed. Spleen was markedly enlarged

Table 2. Percentage of Subjects with Sign and Symptoms of CML disease

\begin{tabular}{lc}
\hline Sign and Symptoms & No (\%) \\
\hline Sleep disturbance & $43(84.31 \%)$ \\
Anxiety & $41(82 \%)$ \\
Depression & $38(74.50 \%)$ \\
Unusual hair loss & $6(11.76 \%)$ \\
Night Sweating & $07(13.72 \%)$ \\
Weight loss & $23(45.09 \%)$ \\
Dry skin & $29(56.86 \%)$ \\
Swelling on Body & $19(37.25 \%)$ \\
Spleen enlargement & $21(41.17 \%)$ \\
Liver enlargement & $19(37.52 \%)$ \\
Blurred vision & $35(64.62 \%)$ \\
\hline
\end{tabular}

Table 3. Clinical Features of the CML Patients Successfully Treated after 12- 48 months by High dose of Imatinib Mesylate (Gleevec) $400-600 \mathrm{mg} / \mathrm{dl}$

\begin{tabular}{lc}
\hline Characteristics & No (\%) \\
\hline Hematological Response & \\
WBC 103/ul & $27(52.94 \%)$ \\
Hemoglobin mg/dl & $30(58.82 \%)$ \\
Platelets 103/ul & $25(49.01 \%)$ \\
Cytogenetic Response [20] cells Ph +/ Ph- & \\
Complete & $3(5.88 \%)$ \\
Partial & $4(7.84)$ \\
Minor & $7(13.52 \%)$ \\
Accelerated to chronic phase & $01(1.96 \%)$ \\
\hline
\end{tabular}




\section{Bibi Tahira et al}

in $41.76 \%$ patients. Spleen size was greater in patients with history of fatigue and sweats. The most common sign of CML was sleeping disturbance, approximately in $84.31 \%$ almost similar to that was anxiety $82 \%$. CML taint patients internally, due to which the patients become very depressed and this depression cause mental disorder in them. Depression was most common in CML patients. $74.50 \%$ patients were depressed due to this malignant disease. Hair loss was found in $11.76 \%$ CML patients. They suffered from this symptom due to body pain, weakness and drug, etc. CML patients complained of sweating were $13.72 \%$. Liver enlargement and blurred vision were also observed in $37.52 \%$ and $64.62 \%$ respectively in CML patients.

Weight loss was found in every age of CML patient. This was due to nausea and vomiting. They were found 41.76\%. CML patients who complained swelling on foot and hands were $37.25 \%$. Their skin looks very dry, hard and was allergic. They had infection in their body in ring form mostly in their chest.

Table 3 presents a summary of hematologic and cytogenetic response rates to $i$ Imatinib mesylate therapy. Response to Imatinib (Glivecis) was usually observed wit 6 months interval after starting therapy. Platelet counts of less than $400 \times 10^{3} / \mathrm{L}$ were observed in 27 , WBC count less than $11 \times 10^{3} / \mathrm{L}$, were observed in 23 cases and hemoglobin values greater than $10 \mathrm{mg} / \mathrm{dl}$ were observed in 27 patients.

Complete cytogenetic response of 51 were observed in 03 (0\% Ph-positive cells), partial 04, (1-5 Ph-positive cells) and minor 07 (6-15 Ph-positive cells) CML patients. Survival was calculated from the time treatment began until death from any cause.

\section{Discussion}

Chronic myeloid leukemia is a malignant disease of clonal stem cell disorder caused by balanced translocation between the long arms of 9, 22 chromosomes, this chromosome referred to as Philadelphia chromosome and is responsible for the production of a protein $\mathrm{BCR}-\mathrm{ABL}$ fusion gene which has a protein kinase activity. The BCRABL gene interfere with white blood cells that making it difficult for the body to fight off infections (QuintasCardama and Cortes, 2006).

Aguayo et al. (2008) reported that the median age of CML patients 55-60 years, were more affected by CML disease but all age groups including children's (10\%) were also affected. Faderel et al. (1999) reported 15 percent adults and 12-30 percent old age 60 years older persons were also affected from CML. 1In this study the median age was found between 25-35. We observed that CML was also accrued from children's to old age persons Table 1 .

In more than $90 \%$ of CML cases, the presence of the Philadelphia chromosome $(\mathrm{Ph})$, which is the product of a balanced translocation between the long arms of chromosomes 9 and 22 is observed (Achkar et al., 2010). Cytogenetic test of $45 \mathrm{CML}$ patients were performed to determine $\mathrm{Ph}$ positive and $\mathrm{Ph}$ negative chromosome. FISH test was performed to identify BCR-ABL gene.

After cytogenetic analysis we observed that 40 (88.88\%) CML patients had Ph positive (9;22) (q34;q11) chromosome and $4(8.88 \%)$ CML showed $\mathrm{Ph}$ negative translocations.

Jaiswal et al. (2003) expressed that CML victims have splenomegaly and hepatomegaly. They have visibly pale bone-marrow and morphological examination of hematopoietic tissues show large proliferation of undifferentiated myeloblasts. CML sufferers with translocation $\mathrm{t}(9 ; 22)$ often show abnormalities in erythrocytes and platelets.

Wiernik (1991) stated that CML can change into fast growing leukemic cells that invade almost any organ in the body. The signs and symptoms of CML patients tend to develop gradually which include easy bruising, tiredness, bleeding, shortness of breath, night sweats, enlarged spleen and liver, and bone pain and fever.

These symptoms like Weight loss 23 (37.25\%), night sweating $7(13.72 \%)$, splenomegaly $21(41.47 \%)$ and hepatomegaly $19(37.52 \%)$, blurred vision $35(64.62 \%)$, depression $38(74.50 \%)$, anxiety $41(82 \%)$ Swelling on Body 19 (37.25\%), and Unusual hair loss 6 (11.76\%) were also significantly observed among CML patients in this study.

Hematological test of all 51 patients were performed. We observed that only four patients of CML had normal WBC counts 4-11 103/ul. Four patients out of 51 had WBC range between 12-20 103/ul, three patients had WBC in between 21-30 10\%/ul, five patients with WBC count in 31$4010^{3} / \mathrm{ul}$, four had WBC count between $41-50 \mathrm{~mm}^{3}$, fifteen patients had WBC between 50-100 103/ul, nine patients had WBC count between 101-300 $\mathrm{mm}^{3}$, two patients WBC count was between301-400 103/ul and five patients had large number of WBC count, above then $40010^{3} / \mathrm{ul}$. And highest WBC $560.210^{3} / \mathrm{ul}$ were identified in this study.

In this study normal PLTs count were observed 150000-400000 in 35 CML patients. The highest platelets count out of $51 \mathrm{CML}$ patients $836000 \mathrm{~mm}^{3}$ were observed. Hemoglobin level observed less than $12 \mathrm{mg} / \mathrm{dl}$ in thirtyfive CML patients and normal hemoglobin above 12 $\mathrm{mg} / \mathrm{dl}$ level was found in sixteen CML patients. Lowest hemoglobin level $5.6 \mathrm{mg} / \mathrm{dl}$ was observed at the time of diagnosis.

Imatinib mesylate (Glivec, Novartis Pharmaceuticals) is an aggressive inhibitor of the BCR-ABL protein-tyrosine kinase. Its therapeutic doses also inhibits tyrosine kinase activity of the platelet-derived growth factor (PDGF) receptor $\beta$ and c-Kit, but does not affect other members of the type III receptor kinase family, such as Flt-3 and Fms (Cohen et al., 2002b; Zangari et al., 2004). In this study all 51 CML patients were successfully treated with Imatinib mesylate which was provided by Sundaymen Hospital, previously some patients were treated with hydroxyurea, but no one got well from use of hydroxyurea tablets.

Initially, enrolled patients treated with orally administered imatinib at daily doses of $400 \mathrm{mg}$ then the availability of phase (chronic or accelerated) this initial daily dose was increased $400 \mathrm{mg}$ to $600 \mathrm{mg}$ which produced complete hematological response in more than $27(52.94 \%)$ patients and complete cryptogenic response achieved in $3(5.88 \%)$ CML patients at 12-18 months therapy. Among all 51 patients only 2 patients died due to allogenic stem cell translocations. 
Out of $51 \mathrm{CML}$ patients 4 were in accelerated phase after 18 months Imatinib therapy 1 shows complete cytogenetic response $(0 \% \mathrm{Ph}+$ cells $)$ and hematological response. And second shows partial cytogenetic but complete hematological response and return to chronic phase.

In conclusion, this study conducted on $51 \% \mathrm{CML}$ patients, it was observed that the number of female patients were more than the number of male patients. This disease was occurred in all ages, from childhood to old age in both male and female patients. The median age was found between 25 to 35 years. The most common symptoms were weight loss, depression, anxiety, spleen enlargement, dry skin. Eye pain and sleep disturbance were common symptoms among all CML patients.

\section{Acknowledgements}

Muhammad Asif is PhD student in BUITEMS under Registration No. 27934 and this study is part of his dissertation towards doctoral degree. Part of this study was supported by BUITEMS, Quetta and King Abdulaziz City for Science and Technology (KACST Strategic Project Number. 12-MED3078-03), Saudi Arabia.

\section{References}

Achkar WA, Wafa A, Ali BY, et al (2010). A rare chronic myeloid leukemia case with Philadelphia chromosome, BCR-ABL e13a3 transcript and complex translocation involving four different chromosomes. Oncol Lett, 1, 797-800.

Aguayo A, Garcia-Alvarez E, Cazares-Ordonez Y, et al (2008). Chronic myeloid leukemia: a clinicoepidemiologic and therapeutic description of a single institution in Mexico City. Clinical Leukemia, 2, 261-6.

Al-Achkar W, Wafa A, Liehr T (2013). A new t (9; 11; 20; 22) (q34; p11.2; q11.21; q11) in a Philadelphia-positive chronic myeloid leukemia case. Oncology Letters, 5, 605-8.

Al-Jamal HA, Jusoh SA, Yong AC, et al (2014). Silencing of suppressor of cytokine signaling-3 due to methylation results in phosphorylation of STAT3 in imatinib resistant BCR-ABL positive chronic myeloid leukemia cells. Asian Pac J Cancer Prev, 15, 4555-61.

Au WY, Caguioa PB, Chuah C, et al (2009). Chronic myeloid leukemia in Asia. Int J Hematol, 89, 14-23.

Chavan D, Ahmad F, Iyer P, et al (2006). Cytogenetic investigation in chronic myeloid leukemia: study from an Indian population. Asian Pac J Cancer Prev, 7, 423-6.

Claussen U, Michel S, Muhlig P, et al (2002). Demystifying chromosome preparation and the implications for the concept of chromosome condensation during mitosis. Cytogenet Genome Res, 98, 136-46.

Cohen MH, Williams G, Johnson JR, et al (2002a). Approval summary for imatinib mesylate capsules in the treatment of chronic myelogenous leukemia. Clin Cancer Res, 8, 935-42.

Cohen MS, Hussain HB, Moley JF (2002b). Inhibition of medullary thyroid carcinoma cell proliferation and RET phosphorylation by tyrosine kinase inhibitors. Surgery, 132, 960-6; discussion 6-7.

Cortes J, Giles F, O'Brien S, et al (2003). Result of high-dose imatinib mesylate in patients with Philadelphia chromosomepositive chronic myeloid leukemia after failure of interferonalpha. Blood, 102, 83-6.

Cowan-Jacob SW, Fendrich G, Floersheimer A, et al (2007).
Structural biology contributions to the discovery of drugs to treat chronic myelogenous leukaemia. Acta Crystallogr D Biol Crystallogr, 63, 80-93.

Faderl S, Talpaz M, Estrov Z, et al (1999). The biology of chronic myeloid leukemia. $N$ Engl J Med, 341, 164-72.

Jabbour E, Kantarjian HM, Jones D, et al (2009). Imatinib mesylate dose escalation is associated with durable responses in patients with chronic myeloid leukemia after cytogenetic failure on standard-dose imatinib therapy. Blood, 113, 2154-60.

Jaiswal S, Traver D, Miyamoto T, et al (2003). Expression of BCR/ABL and BCL-2 in myeloid progenitors leads to myeloid leukemias. Proceed Nat Acad Sci, 100, 10002-7.

Koshiyama DB, Capra ME, Paskulin GA, et al (2013). Cytogenetic response to imatinib treatment in Southern Brazilian patients with chronic myelogenous leukemia and variant Philadelphia chromosome. Ann Hematol, 92, 185-9.

La Starza R, Testoni N, Lafage-Pochitaloff M, et al (2002). Complex variant Philadelphia translocations involving the short arm of chromosome 6 in chronic myeloid leukemia. Haematologica, 87, 143-7.

Quintas-Cardama A, Cortes JE (2006). Chronic myeloid leukemia: diagnosis and treatment. Mayo Clin Proc, 81, 973-88.

Sawyers CL (1999). Chronic myeloid leukemia. N Engl J Med, 340, 1330-40.

Sessarego M, Fugazza G, Bruzzone R, et al (2000). Complex chromosome rearrangements may locate the bcr/abl fusion gene sites other than 22q11. Haematologica, 85, 35-9.

Simons A, Shaffer LG, Hastings RJ (2013). Cytogenetic nomenclature: changes in the ISCN 2013 compared to the 2009 edition. Cytogenet Genome Res [Epub ahead of print].

Usmani SZ, Yunus SA, Jamal Y (2009). Overview of chronic myeloid leukemia patients in Pakistan in the pre-imatanib era. Asian Pac J Cancer Prev, 10, 1039-40.

Wiernik PH (2003). Neoplastic diseases of the blood, Cambridge University Press.

Ye YX, Zhou J, Zhou YH, et al (2014). Clinical significance of BCR-ABL fusion gene subtypes in chronic myelogenous and acute lymphoblastic leukemias. Asian Pac J Cancer Prev, 15, 9961-6.

Zangari M, Anaissie E, Stopeck A, et al (2004). Phase II study of SU5416, a small molecule vascular endothelial growth factor tyrosine kinase receptor inhibitor, in patients with refractory multiple myeloma. Clin Cancer Res, 10, 88-95. 